\title{
Synthesis and Antibacterial Activity of 2-Monolaurin
}

\author{
FEBRI ODEL NITBANI ${ }^{1,3}$, JUMINA ${ }^{1 *}$, DWI SISWANTA ${ }^{1}$, \\ ETI NURWENING SHOLIKHAH ${ }^{2}$ and DHINA FITRIASTUTI ${ }^{4}$
}

\author{
'Department of Chemistry, Faculty of Mathematics and Natural Sciences, \\ Universitas Gadjah Mada, Yogyakarta, Indonesia. \\ 2Department of Pharmacology and Therapy, Faculty of Medicine, \\ Universitas Gadjah Mada Yogyakarta, Indonesia. \\ ${ }^{3}$ Department of Chemistry, Faculty of Science and Engineering, \\ Universitas Nusa Cendana, Kupang, Indonesia. \\ ${ }^{4}$ Department of Chemistry, Faculty of Mathematics and Natural Sciences, \\ Universitas Islam Indonesia, Yogyakarta, Indonesia. \\ ${ }^{*}$ Corresponding author E-mail: jumina@ugm.ac.id
}

http://dx.doi.org/10.13005/ojc/320632

(Received: November 04, 2016; Accepted: December 08, 2016)

\begin{abstract}
Ethanolysis reaction triglyceride from coconut oil using enzyme from lipozyme $T L I M$ has produced a mixture of 2-mono acyl glycerol. Product 2-mono acyl glycerol with a yield of $35 \%$ has been achieved in the ratio by weight of triglyceride from coconut oil to dry ethanol is $1: 4$, temperature of $35^{\circ} \mathrm{C}$, the number of lipozyme $T L I M 5 \%(\mathrm{w} / \mathrm{w})$ of the total weight of the reactants and reaction time 12 hours. The 2-monolaurin compound with a purity of $100 \%$ and a yield of $30,1 \%$ successfully separated from 2-mono acyl glycerol. The 2-monolaurin compound inhibited the growth of Staphylococcus aureus and Bacillus cereus at a concentration of $2500 \mathrm{ppm}$ each with a diameter of inhibitory zone 13.75 and $10.44 \mathrm{~mm}$. Product mixture 2-mono acylglycerol inhibited the growth of Staphylococcus aureus and Bacillus cereus respectively at concentrations of 100 and 1000 ppm with a diameter of inhibitory zone 10.32 and $10.83 \mathrm{~mm}$.
\end{abstract}

Keywords: Coconut oil, Ethanolysis, Monoacylglycerol, Monolaurin, Antibacterial.

\section{INTRODUCTION}

Monoacylglycerol is a class of lipid compounds which have an important role in the food, cosmetics and pharmaceutical industry ${ }^{1}$. Monoglyceride containing medium chain fatty acids has been reported to play important role in medical and nutritional application ${ }^{2}$. One of monoacylglycerol from coconut oil-based material is monolaurin. Based on their chemical structure, monolaurin can present as compound 1-monolaurin and 2-monolaurin. To be sure that a monolaurin certainly contains two hydroxyl groups and one group lauryl. Thus monolaurin is a non-ionic surfactant containing hydrophilic and hydrophobic groups in the molecule at the same time. 
Babies and children depend on monolaurin for the development of the immune system and their ability to resist infections due to bacteria ${ }^{3}$. Monolaurin are essential fats contained in breast milk ${ }^{4}$. The antimicrobial activity of monolaurin is a broad-spectrum antimicrobial activity. Monolaurin showed have antifungal activity against Aspergillus sp, Penicillium sp., Cladosporium sp., Fusarium sp., Alternaria sp., Candida albicans, Fonsecaea pedrosoiand Cryptococcus neoformans ${ }^{5}$. Monolaurin also have antiviral activity and works actively to dissolve lipids and phospholipids in the hulls of viruses that cause damaged of viral envelope ${ }^{6}$.

The reaction of lauric acid and glycerol using catalysts lipozyme IM-20 has produced 1-monolaurin compound ${ }^{7}$. The obtained 1-monolaurin compound reach a conversion rate of $80 \%$ and there are byproducts like dilaurin and trilaurin. Synthesis of 1 -monolaurin with a yield of $31.05 \%$ has been produced through esterification reaction of glycerol with lauric acid and sulfuric acid catalyst ${ }^{8}$. Compound 1-monolaurin produced has sufficiently strong antibacterial activity against Staphylococcus aureus than byproducts dilaurin. Both the results of this study indicate that the type of monolaurin synthesized is 1-monolaurin and derived from the basic material of lauric acid. In addition, the reaction process using sulfuric acid catalyst which is toxic and enzymes Lipozyme IM-20 which was very expensive.

This research is aimed to synthesize the compound 2-monolaurin directly from coconut oil and test its antibacterial activity against bacteria Staphylococcus aureus, Salmonella thypimorium, Bacillus cereus, and Escherichia coli. The use of coconut oil is the austerity measures in the synthesis of 2-monolaurin. Coconut oil are abundant, cheap and does not involve costly lauric acid isolation. The catalyst used is Lipozyme TL IM which is available at relatively cheap price when compared to other lipase enzyme produced by Novozym.

\section{EXPERIMENTAL}

\section{Materials}

Materials used in this experiment were neutralized coconut oil (acid number $<1$ ) ${ }^{9}, \mathrm{Na}_{2} \mathrm{CO}_{3}$, Lipozyme TL IM, n-hexane, ethanol, chloroform, acetone, methanol, DMSO and distilled water. All the chemicals used were purchased by Merck except distilled water and coconut oil.

\section{Instruments}

Chromatogram and mass spectra were measured on Mariner LC-MS using C-18 column $(15 \times 1 \mathrm{~mm})$ and injected volume of $2 \mu \mathrm{L}$. The mobile phase is methanol with rate flow of $0.1 \mathrm{~mL} / \mathrm{min}$.

\section{Procedure}

\section{Synthesis and Purification of 2-Monolaurin}

A mixture of $750 \mathrm{mg}$ of neutralized coconut oil and $3 \mathrm{~g}$ of dry ethanol were placed in $25 \mathrm{~mL}$ of falcon. As much as $375 \mathrm{mg}$ of Lipozyme TL IM (10\% $(\mathrm{w} / \mathrm{w})$ of total reactant) was placed in the mixture of reaction and the vial was placed in a water bath shaker incubator. Reaction was done at $55^{\circ} \mathrm{C}$ for 6 $\mathrm{h}$. The result of reaction was filtered to separate the product from enzyme. The filtrate was dissolved in hydroalcoholic solution (ethanol:water= 8:2) as much as 9 times of filtrate volume. The mixture was then extracted with n-hexane. Monoacylglycerol which dissolved in hydroalcoholic phase was evaporated and weighed. The product was analyzed using LC-MS. The optimization of reaction was done at temperature of $45^{\circ} \mathrm{C}$ and $35^{\circ} \mathrm{C}$. The temperature that produces higher yield of reaction was chosen as optimal temperature for optimizing total of enzyme and time reaction. Variation of used total enzyme was 5,15 and $20 \%$ (w/w) of total mass of reactant (oil and dry ethanol). Furthermore, the variation of time reaction was 12,18 and 24 hours.

The product of variation of temperature, total of enzyme and time reaction that contain the highest percentage of monoacylglycerol was then purified using TLC preparative. A total of $1 \mathrm{~g}$ of the product 2-mono acyl glycerol dissolved in $5 \mathrm{~mL}$ of acetone and then spotted $3 \mathrm{~cm}$ from the lower bound on silica TLC plate Preparative size of $20 \times 20 \mathrm{~cm}$. Eluent included in the developer vessel is chloroform: acetone: methanol (9.5: 0.45: 0.05) 30 $\mathrm{mL}$. Plate silica containing monoacylglycerol samples of coarse subsequently included in the developer vessel containing the eluent. Furthermore, there is a separation until the eluent reaches the upper limit that has been marked. Noda looked dominant in UV light at a low retention time later extracted and dissolved in $12 \mathrm{~mL}$ of acetone and filtering the silica. The solid product obtained by evaporation of 
the solvent acetone and recrystallized in n-hexane. Products are weighed and measured melting point. Monolaurin solids were then analyzed using Liquid Chromatography-Mass Spectrometer (LC-MS).

\section{Antibacterial Assay}

The composition of media for bacteria such as Nutrient Agar consists of $5 \mathrm{~g}$ of peptone, $3 \mathrm{~g}$ of beef extract and $15 \mathrm{~g}$ of agar were dissolved in $1 \mathrm{~L}$ of distilled water and then sterilized at a temperature of $121^{\circ} \mathrm{C}$ for 15 minutes at 15 psi. On the surface of the cup, $50 \mathrm{~mL}$ of $1.8 \%$ agar solution was poured. After solidified, the wells are made by putting a ring diameter of $1 \mathrm{~cm}$ and then the surrounding poured $50 \mathrm{~mL}$ of media for pathogenic bacteria that have been added $1 \%$ culture of pathogens such as Staphylococcus aureus FNCC 0047, Bacillus cereus FNCC 0057, Escherichia coli FNCC 0091 and Salmonella typhimurium FNCC 157. After solidified, in wells added $150 \mathrm{iL}$ of test compound is 2-monoacylglycerol and 2-monolaurin each with a concentration of 500, 1000, 2500, 5000 and 10000 ppm with the negative control DMSO (99.9\%). Then for the positive control used tetracycline at a concentration of $100 \mathrm{ppm}$. The incubation period for 24 hours and the next measurement of inhibitory zone. Clear zone with a size of more than $2 \mathrm{~mm}$ on the surrounding wells indicated as positive inhibition. The diameter of inhibition zone formed was measured by using a caliper to determine the effectiveness of antibacterial. Inhibition zone was measured by reducing the overall diameter (disk + zone of inhibition) diameter disk.

\section{RESULT AND DISCUSSION}

Monolaurin consisting of compound 1-monolaurin and 2-monolaurin. This classification is based on the location of lauryl groups on the carbon skeleton compound glycerol. When lauryl group located at position $\mathrm{C} 1$ atom of the glycerol, it will produce the 1-monolaurin compound. If lauryl group attached to the glycerol $\mathrm{C} 2$ atom, it will produce the 2-monolaurin Compound.

One of the paths that can be taken to produce 2-monolaurin of coconut oil triglycerides is through alcoholysis reaction using specific lipase enzyme catalyst $(s n-1,3)$. Alcohol is selected as a reactant in the process of lysis of the triglyceride from coconut oil is ethanol. Methanol avoided as a reactant in this ethanolysis reaction, because the toxic nature caused by methanol in the reaction product.

Coconut oil is used in the ethanolysis reaction is coconut oil neutral ever undertaken by Nitbani et al., (2016). Triglyceride from coconut oil is purified from free fatty acids and other impurities through the neutralization process using a weak alkaline solution. Triglyceride coconut oil which has 3 acyl groups will be broken down by ethanol in the position of the acyl group 1 and 3 because of the activity of the catalyst of lipase TL IM. Because of lauric acid is a major component in triglyceride of coconut oil, it can be ascertained that the products of ethanolysis will be dominated by the compound

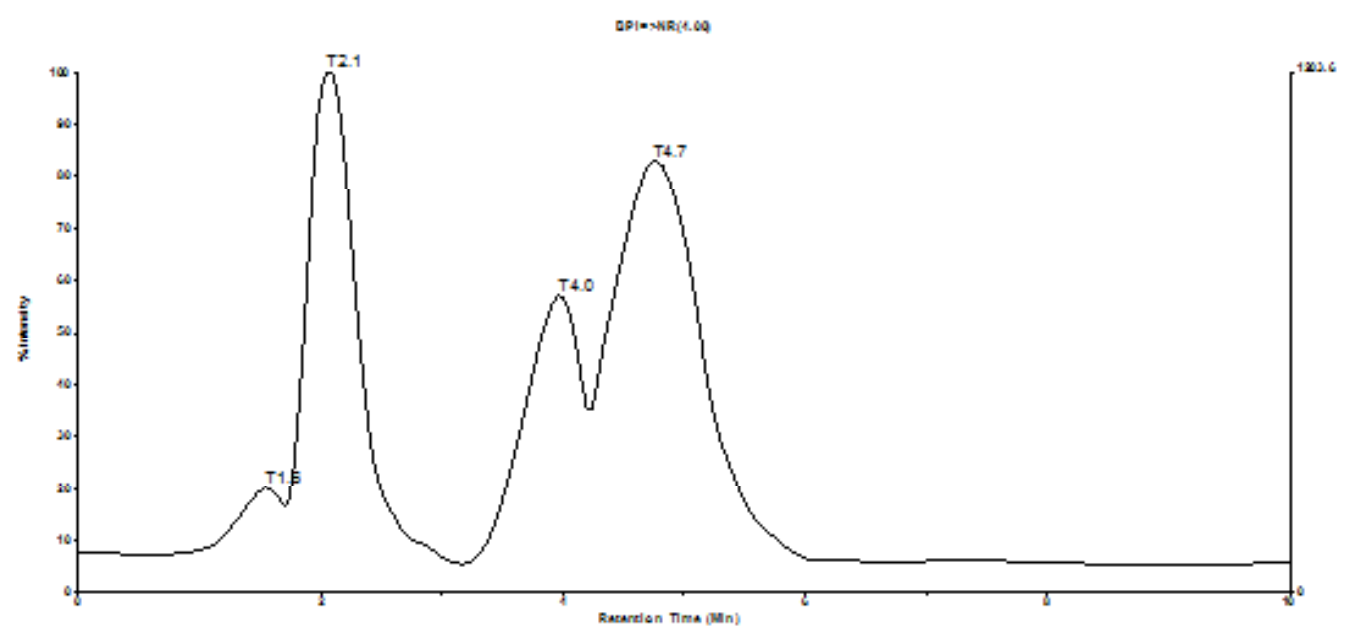

Fig. 1: Chromatogram of ethanolysis reaction product at $\mathrm{T}=55^{\circ} \mathrm{C}$ 
2-mono acyl glycerol from the compound of 2-monolaurin. Isolation of 2-monolaurin product has been done using a solution of ethanol-water at a ratio of 8:2. Product 2-monolaurin is suspected dissolve well in this hydroalcoholic solution.

Ethanolysis reaction product of coconut oil triglycerides in ethanol-water phase in the form of a white solid with a yield of $9.13 \%$. This product contains a mixture of the compound 2-mono acyl glycerol (2-MAG) include 2-monolaurin. Product mix of two monoacylglycerol from coconut oil is produced under the reaction conditions, namely the ratio by weight of triglyceride of coconut oil or neutralized coconut oil to dry ethanol is $1: 4$, the amount of enzyme lipozyme TL IM is $10 \%(\mathrm{w} / \mathrm{w})$ of the total weight of the reactants, reaction temperature $55^{\circ} \mathrm{C}$, a reaction time of 6 hours, the number of hydroalcoholic solution is 9 times the volume of the reaction product filtrate, and the number of n-hexane is 3 times the volume of used hydroalcoholic solution.

To detect that the hydroalcoholic phase containing the compound 2-monolaurin as a kind of 2-monoacylglycerol mayor of triglyceride from coconut oil ethanolysis result, the product then analyzed using LC-MS. Chromatogram analysis result is presented in Figure 1. The data in Figure 1 shows the presence of four peaks with different retention times.

Mass spectra peak with a retention time of 2.1 minutes suspected compound 2-monolaurin is presented in Figure 2. This mass spectra showed the presence of the fragment $\mathrm{M}+\mathrm{H}=275.25$ which is the molecular weight of the compound 2-monolaurin is $274 \mathrm{~g} / \mathrm{mol}$. Another fragment which appeared as a supporter, namely the fragment $M+23=297.34$ and fragments $2 \mathrm{M}+23=571.71$.

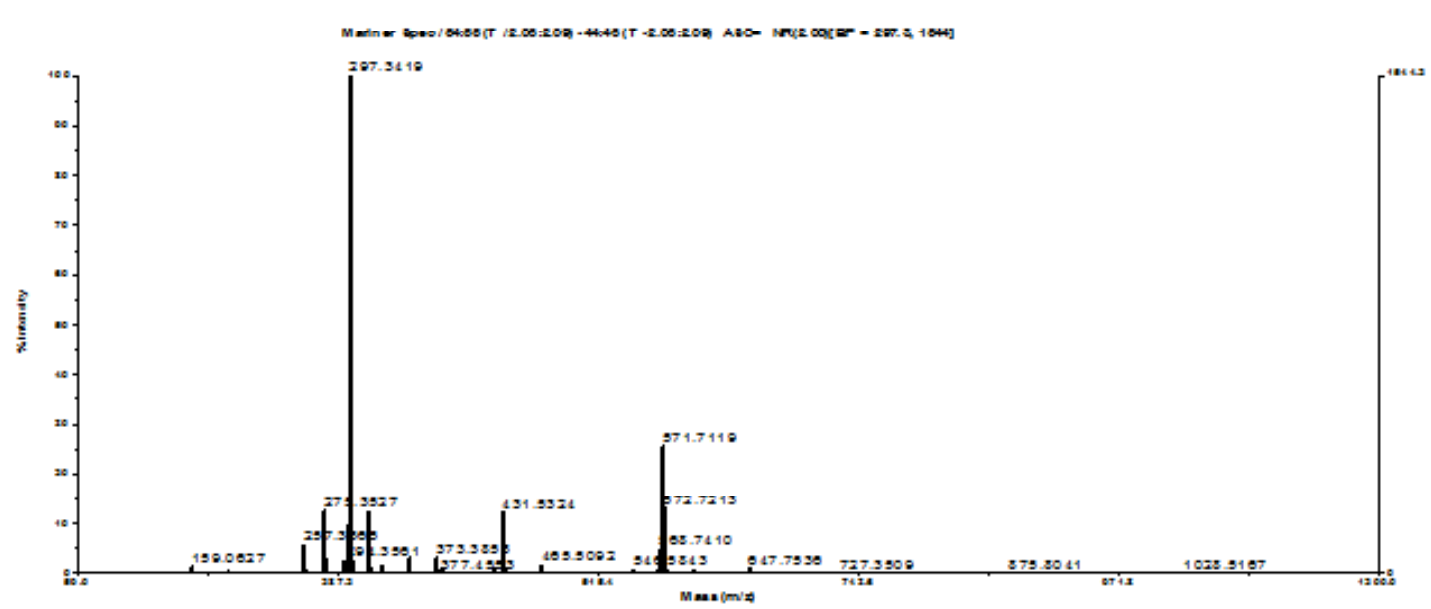

Fig. 2: Mass Spectrum of peak at $t_{R}=2.1 \mathrm{~min}$

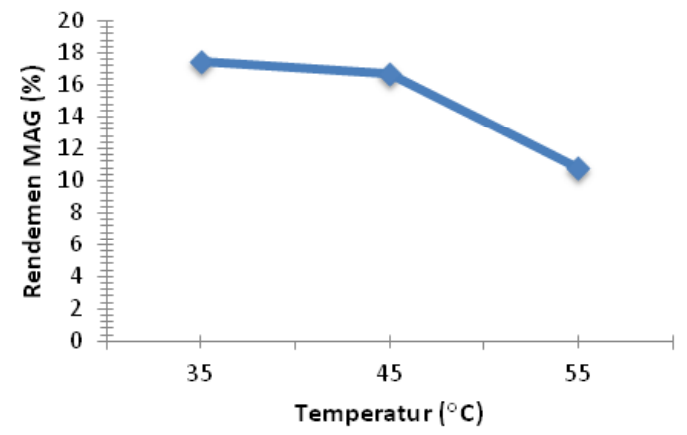

Fig. 3: Effect of temperature vs yield of 2-MAG

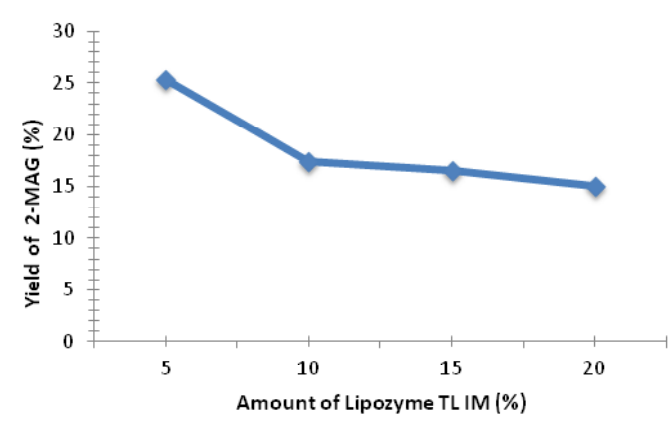

Fig. 4: Effect of the amount of Lipozyme TL IM vs yield of 2-MAG 
It can be concluded that the hydroalcoholic phase of ethanolysis product from triglyceride coconut oil reacted at a temperature of $55^{\circ} \mathrm{C}$ containing the compound 2-monolaurin with $38.44 \%$ purity. Therefore, there will be a reaction optimization ethanolysis triglyceride of coconut oil using enzymes Lipozyme TL IM at another temperature variations, as

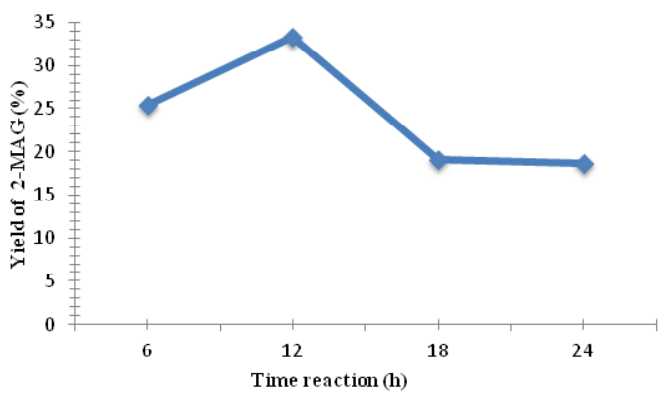

Fig. 5: Effect of time reaction vs yield of 2-MAG well as to the amount of lipase enzyme and reaction time. To determine the effect of temperature on the amount or yield ethanolysis product of triglyceride from coconut oil reaction is carried out at another temperature variations at a temperature of $45^{\circ} \mathrm{C}$ and $35^{\circ} \mathrm{C}$. Temperature greatly affects the catalytic activity of the enzyme of Lipozyme TL IM that is used as a catalyst in the reaction ethanolysis triglyceride of coconut oil.

Facts about the effect of temperature on the enzyme activity Lipozyme TL IM can be explained as follows that the enzyme is a protein that naturally have a three-dimensional structure formed by chemical bonding types such as hydrogen and Van der Walls bonding. The integrity of three dimension shape or form conformation of the enzyme is very important in the catalytic activity of the enzyme and determine its specific function. The conformation of the enzyme survives at a given temperature. Temperature change

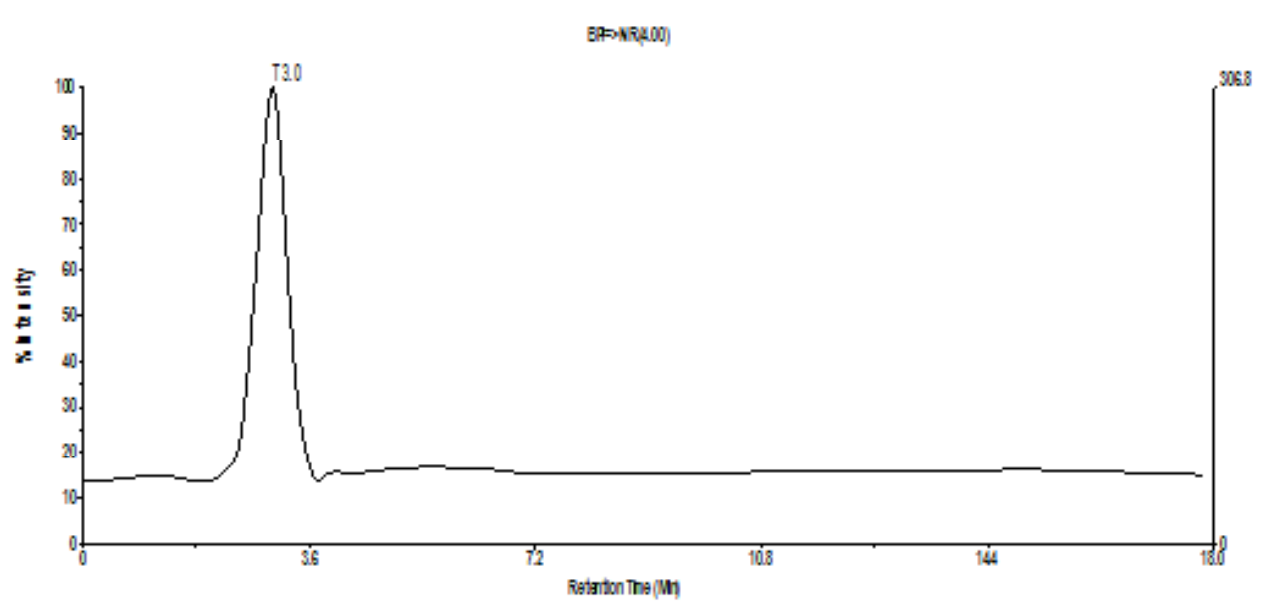

Fig. 6: Chromatogram of 2-monolaurin

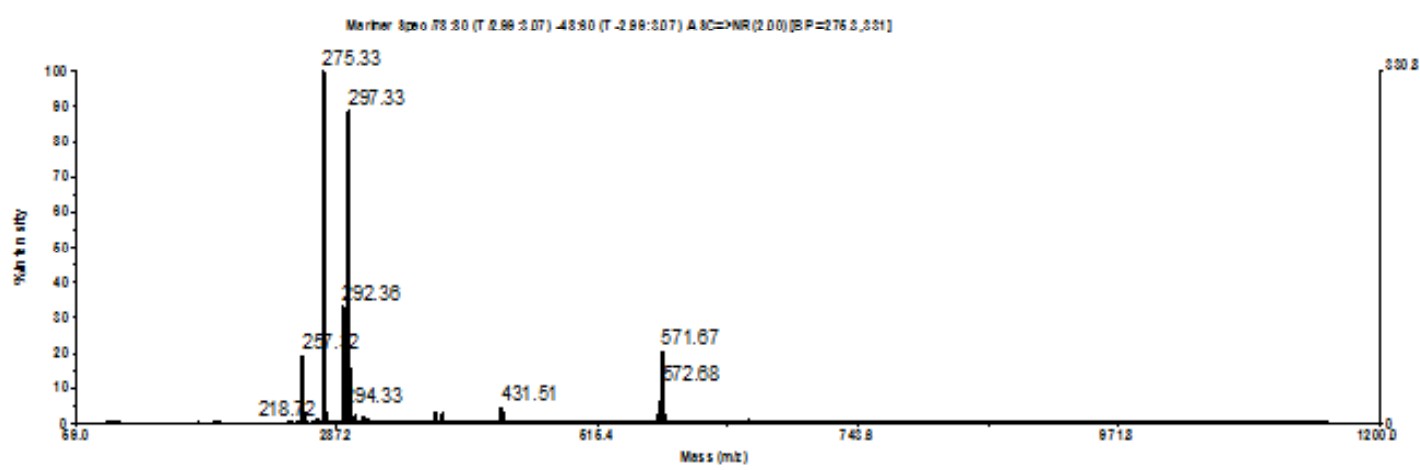

Fig. 7: Mass spectrum of 2-monolaurin 
can affect the enzyme intramolecular bonds that can change shape conformation of the enzyme. With these changes will lead to changes in the catalytic properties or activity of the enzyme.

The graph in Figure 3 shows the results of 2-monoacylglycerol yield of optimization at various temperatures. Data graph shows that the yield of the product 2-monoacylglycerol high of $18.21 \%$ is achieved at a temperature of $35^{\circ} \mathrm{C}$. This result was achieved when the reaction conditions with some fixed variables except the variable temperature. Thus it can be said that the optimum temperature lipozyme TL IM enzyme catalyzes ethanolysis triglyceride of coconut oil is $35^{\circ} \mathrm{C}$. Temperature $35^{\circ} \mathrm{C}$ then used as a reference as reaction temperature in the optimization of ethanolysis triglyceride of coconut oil advanced i.e. varying the amount of enzyme lipozyme TL IM and reaction time.

Reaction ethanolysis of triglyceride of coconut oil was performed at the ratio fixed weight of the reactants, reaction time 6 hours, reaction temperature $35^{\circ} \mathrm{C}$ but the amount of enzyme lipozyme TL IM was varied of $5 \%, 15 \%$ and $20 \%$ $(\mathrm{w} / \mathrm{w})$ of total weight of the reactants. By using the same conditions i.e extraction technique, the amount of ethanol-water solution and the amount of n-hexane, the white solid product obtained of each treatment with a different final weight. Graph effect the amount of TL IM lipozyme to yield of 2-monoacylglycerol can be seen in Figure 4. Data graph in Figure 4 shows that the highest yield of 2-monoacylglycerol product obtained when the amount of TL IM lipozyme by $5 \%$ $(\mathrm{w} / \mathrm{w})$ of total reactant with yield of $26 \%$. In a condition where the amount of enzyme lipozyme TL IM is already the maximum and the amount of substrate is fixed then increasing the amount of enzyme will not increase the reaction rate which proportional to the increase in the amount of products. This is because the enzymes have the active site as a venue for the catalytic activity and its capacity is also limited. If the active site is already filled with the substrate then increasing the number of substrates will not increase the rate of the reaction because of the reaction rate is already the maximum.

Table 1: Diameter of zone inhibition

\begin{tabular}{|c|c|c|c|c|c|c|}
\hline \multirow[t]{2}{*}{ Sample } & \multirow[t]{2}{*}{ Bacteria } & \multicolumn{5}{|c|}{ Diameter of zone inhibition (mm) } \\
\hline & & $\begin{array}{c}100 \\
\text { ppm }\end{array}$ & $\begin{array}{l}1000 \\
\text { ppm }\end{array}$ & $\begin{array}{l}2500 \\
\text { ppm }\end{array}$ & $\begin{array}{l}5000 \\
\text { ppm }\end{array}$ & $\begin{array}{c}10000 \\
\text { ppm }\end{array}$ \\
\hline \multirow[t]{4}{*}{ 2-monoacylglycerol (A) } & S. aureus & 10.32 & 21.57 & 38.73 & 40.84 & 42.02 \\
\hline & E. coli & 0 & 0 & 0 & 0 & 0 \\
\hline & S. typhimuirium & 0 & 0 & 0 & 0 & 0 \\
\hline & B. cereus & 0 & 10.83 & 30.90 & 36.73 & 39.23 \\
\hline \multirow[t]{4}{*}{ 2-monolaurin (B) } & S. aureus & 0 & 0 & 13.75 & 13.98 & 14.35 \\
\hline & E. coli & 0 & 0 & 0 & 0 & 0 \\
\hline & S. typhimuirium & 0 & 0 & 0 & 0 & 0 \\
\hline & B. cereus & 0 & 0 & 10.44 & 38.48 & 40.59 \\
\hline \multirow[t]{4}{*}{ Tetracycline $(\mathrm{K}+)$} & S. aureus & 33 & & & & \\
\hline & E. coli & 25 & & & & \\
\hline & S. typhimuirium & 25 & & & & \\
\hline & B. cereus & 36 & & & & \\
\hline \multirow[t]{4}{*}{ DMSO (K-) } & S. aureus & 0 & & & & \\
\hline & E. coli & 0 & & & & \\
\hline & S. typhimuirium & 0 & & & & \\
\hline & B. cereus & 0 & & & & \\
\hline 1-monolaurin* & S. aureus & 7 & 8.5 & 8.5 & 9 & 9.5 \\
\hline
\end{tabular}

*Widiyarti et al., 2009 (Indonesian Journal of Chemistry) 
The reaction time can affect the yield of coconut oil triglyceride ethanolysis product using an enzyme catalyst lipozyme TL IM. In the fixed variable conditions, temperature $35^{\circ} \mathrm{C}$ and the amount of lipase $5 \%(\mathrm{w} / \mathrm{w})$ of total weight of the reactants, then the variation of reaction time is 12,18 , and 24 hours. Effect of reaction time on the yield of 2-monoacylglycerol are presented in Figure 5. This graph shows that the yield of 2-monoacylglycerol products reached $35 \%$ at 12 hours reaction time.

Based on optimization data, it can be concluded that coconut oil triglyceride ethanolysis reaction may produce a mixture of 2-monoacylglycerol in the form of a white solid after partitioned in an ethanol-water solution (8:2) and washing using $n$-hexane. The highest yield a mixture of 2-monoacylglycerol achieved under reaction conditions i.e the ratio by weight of triglyceride of coconut oil or neutralized coconut oil and dry ethanol (1: 4), a temperature of $35^{\circ} \mathrm{C}$, the amount of lipozim TL IM $(5 \%(w / w))$ of total weight of the reactants) and reaction time 12 hours.

Qualitative analysis using Thin Layer Chromatography (TLC) with eluent chloroform, acetone and methanol (9.5: 4.5: 0.05), performed on products with maximum yield. Profile stains generated such a large stain at Rf of 0.45 and there are also two small stains with long retention time. The distance between suspected stain compound 2-monolaurin quite apart from stain of impurity so it is easy to be separated by Thin Layer Chromatography Preparative. Products successfully separated by recrystallization using $n$-hexane in the form of a white solid with yield $30,1 \%$.

The purity of the compound 2-monolaurin proven through the results of the analysis using LC-
MS. The chromatogram presented in Figure 6 shows that there is only one peak, which means that the analyzed compound has a purity of $100 \%$.

The truth that the peaks in the chromatogram are the compound 2-monolaurin can be confirmed by the mass spectral data (Figure 7). The presence of fragment peaks $\mathrm{M}+\mathrm{H}=275.33$ already showing the molecular weight of the compound 2-monolaurin i.e $274 \mathrm{~g} \mathrm{/} \mathrm{mol.} \mathrm{The} \mathrm{other} \mathrm{supporting} \mathrm{peaks} \mathrm{are}$ the presence of fragment of $M+23=297,33$ and fragment of $2 \mathrm{M}+23=571,67$.

All appeared fragments showing that a single peak in the chromatogram is 2-monolaurin compound which has a chemical structure at Figure 8.

Data and facts of this analysis show that the compound 2-monolaurin can be synthesized from coconut oil triglyceride or neutralized coconut oil through lysis reaction using ethanol. It is strongly believed that the resulting product is a compound of 2-monolaurin not 1-monolaurin (Figure 9) because it uses specific lipase enzyme sn-1,3 from Thermomyces lanuginosus Immobilized (TL IM) which only removing the acyl group at position $\mathrm{C}_{1}$ and $\mathrm{C}_{3}$ of triglyceride of coconut oil.

\section{Antibacterial Test}

Test of antibacterial activity in vitro was conducted to determine the ability of a compound to inhibit the growth of test bacteria. Inhibition value measured by the value of the inhibition area. Samples tested compound is a mixture of 2-monoacylglycerol product $(A)$ which is the result of ethanolysis of triglyceride coconut oil in the treatment with the highest yield. Another test sample $(B)$ is 2-monolaurin (purified from product $A$ ). Bacteria<smiles>CCCCC(C)C</smiles>

Fig. 8: Chemical Structure of 2-monolaurin 
test used is Staphylococcus aureus, Salmonella thypimorium, Bacillus cereus, and Escherichia coli. Antibacterial test results in the form of size of diameter of inhibition zone against various test bacteria that is used in Table 1.

The data in Table 1 showed that both a mixture of 2-monoacylglycerol $(A)$ and 2-monolaurin (B) was not active to inhibit bacterial growth Salmonella thypimorium and Escherichia coli. The mix product of 2-monoacylglycerol (A) has a good inhibitory to bacteria Staphylococcus aureus and also Bacillus cereus. The higher of concentration sample $A$ thus the diameter inhibition zone will larger even though still low compared to tetracycline (100 ppm) of the same bacteria.

Compound 2-monolaurin (B) has a good inhibition to bacteria Staphylococcus aureus and also Bacillus cereus at a concentration of 2500 ppm. For Staphylococcus aureus bacteria, the compound 2-monolaurin (B) showed the values inhibition area far exceeds the value of the area inhibition compound 1-monolaurin at a concentration of 2500 ppm. Based on the value of the inhibition area, it can be concluded that the compound 2-monolaurin have inhibitory larger or more active than compound 1-monolaurin in inhibiting bacterial growth, especially Staphylococcus aureus. Compound 2-monolaurin (B) also actively inhibits bacterial growth Bacillus cereus at a concentration of 2500 ppm.
Overall, it can be concluded that the product A was more active than sample B in inhibiting the growth of bacteria Staphylococcus aureus and Bacillus cereus. Sample A containing a mixture of 2-monoacylglycerol from 2-monolaurin and other monoacylglycerol from fatty acid fractions in coconut oil. Those impurities in sample A also contributed to inhibiting the growth of bacteria. This is proven since when the compound of 2-monolaurin $(B)$ can be isolated from the sample $A$, the inhibitory effect on the same test bacteria is decreased.

\section{CONCLUSION}

1. The compound 2-monolaurin with a purity of $100 \%$ can be synthesized from the ethanolysis reaction of triglyceride coconut oil or neutralized coconut oil using a catalyst lipozyme $T L I M$, followed by purification by Thin Layer Chromatography Preparative.

2. A mixture of 2-monoacylglycerol produced from hydroalcoholic fraction is able to inhibit the growth of bacteria Staphylococcus aureus and Bacillus cereus.

3. Compound 2-monolaurin is able to inhibit the growth of bacteria Staphylococcus aureus and Bacillus cereus at a concentration of 2500 ppm.

\section{REFERENCES}

1. Nitbani, F.O.; Jumina; Siswanta, D.; Sholikhah, E.N., Int. J. Pharm. Sci. Rev. Res., 2015, 35 (1), 126-136

2. Nitbani, F.O.; Jumina; Siswanta, D.; Sholikhah, E.N., Int. J. Pharm. Sci. Rev. Res., 2016, 39 (1), 74-80

3. Hierholzer, J.C.; and Kabara, J.J.; J. Food Saf., 1972; 4, 1-12

4. Hedge, B.M.; Journal Indian Academy of Clinical Medicine, 2006, 7(1): 16-19

5. Esquenazi, D.; Wigg, M.D.; Miranda, M.M.; Rodrigues, H.M.; Tostes, J.B; and Rosental, S.;, Res. Microbiol., 2002, 153 (10), 647652
6. Arora, R.; Chawla, R.; Marwah, R.; Arora, P.; Sharma, R.K.; and Khaushik, V.; EvidBased Complement Alternat Med, 2011, doi: $10.1155 / 2011 / 586506$

7. Langone, M.A.P.; Dee Abreu, M.E.; Rezende, M.D.C; Sant'anna, G.L.J.R.;, Appl. Biochem. Biotechnol., 2002, 98-100

8. Widiyarti, G.; Hanafi, M.; Soewarso, W.P.; 2009, 9 (1), 99-106

9. Nitbani, F.O.; Jumina, Siswanta, D.; Sholikhah, E.N.;, Procedia Chemistry, 2016, 18, 132140 